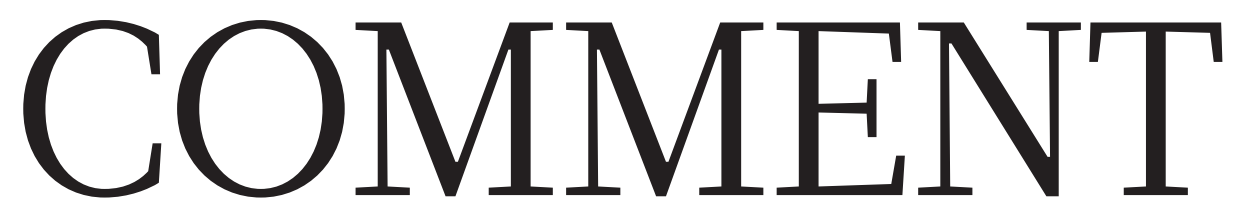

MARINE CONSERVATION A broader concept for protecting ocean reserves $\mathbf{p . 3 9 9}$
PHYSICS How the discovery of cosmic rays a century ago seeded particle physics $\mathbf{p . 4 0 0}$

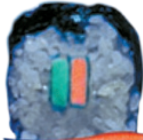

EXHIBITION Transgenic sushi and the future of food $\mathbf{p . 4 0 4}$
OBITUARY Renato Dulbecco, elegant pioneer of cancer biology $\mathbf{p . 4 0 8}$

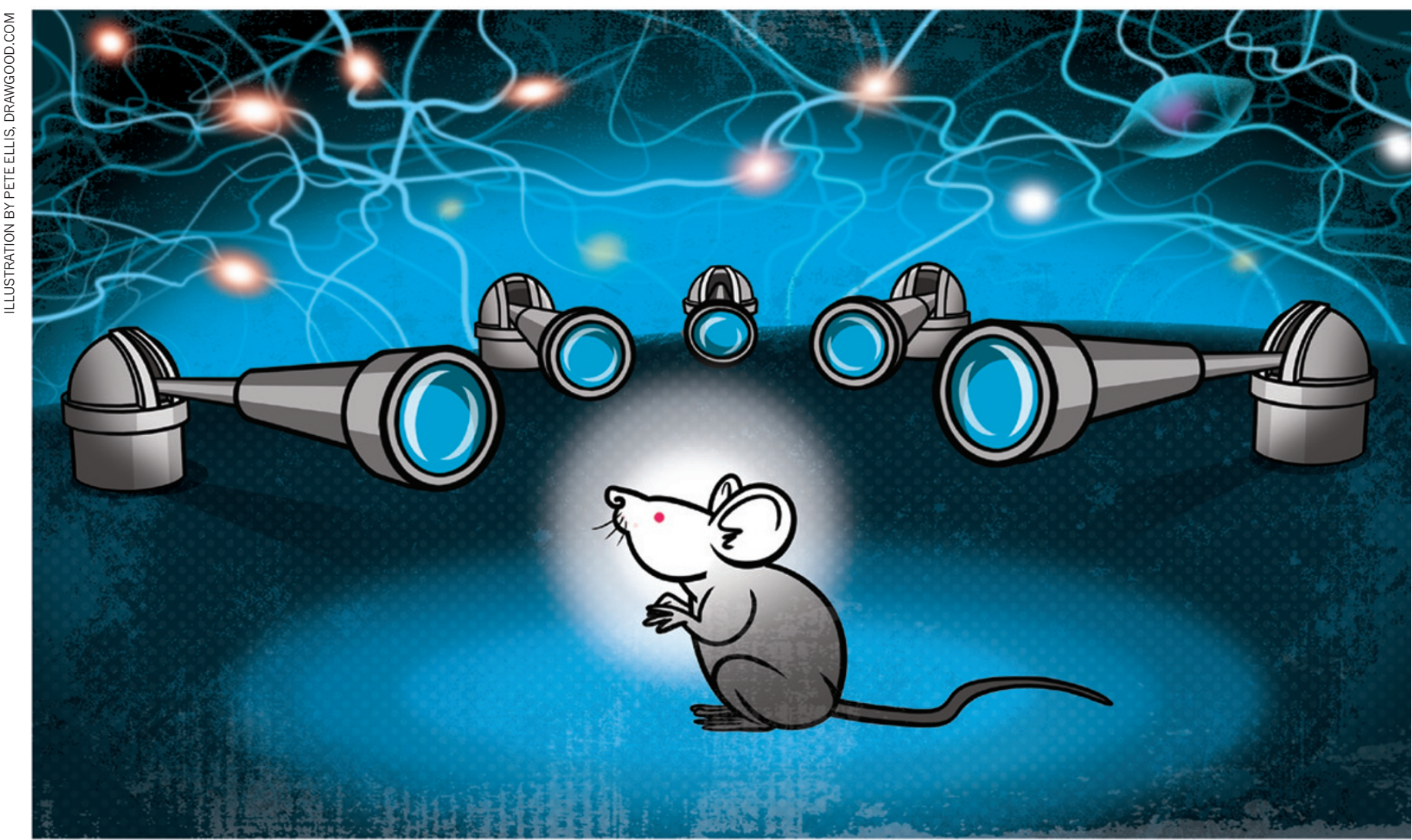

\title{
Observatories of the mind
}

An ambitious project to map the mouse brain at the Allen Institute for Brain Science is a huge undertaking that may unify neuroscience, argue Christof Koch and R. Clay Reid.

$\mathrm{N}$

euroscience is a splintered field. Some 10,000 laboratories worldwide are pursuing distinct questions about the brain across a panoply of spatio-temporal scales and in a dizzying variety of animal species, behaviours and developmental time-points. At any large neuroscience meeting, one is struck by the pace of discovery, with 50,000 or more practitioners heading away from each other in all directions, in a sort of scientific Big Bang.

Although this independence is necessary, it has prevented neuroscience from entering a more mature phase, which would involve developing common standards and collaborative projects. Neurophysiologists are more likely to use each other's toothbrushes than each other's data and software; physiological results are hoarded and rarely made accessible online; molecular compounds and transgenic animals are shared only after publication. All of this has made comparisons across laboratories difficult and has slowed progress.

At the Allen Institute for Brain Science in Seattle, Washington, we and our colleagues are initiating an experiment in the sociology of neuroscience - a huge endeavour that will involve several hundred scientists, engineers and technicians at the institute. Philanthropist Paul G. Allen, who founded the institute in 2003, has pledged US $\$ 300$ million for the first four years of an ambitious ten-year plan that will accelerate progress in neuroscience, bringing his total commitment so far to $\$ 500$ million. Our goal is to attract the best young scientists and build a series of 'brain observatories', with the aim of identifying, recording and intervening in the mouse cerebral cortex, the outermost layer of the brain. Unlike the telescopes that peer at remote events in space 
$\checkmark$ and time, our instruments will track the flow of information in complex, interbraided neural circuits within a layer of tissue one millimetre thick.

The goal is to synthesize knowledge from all relevant disciplines - from genomics, anatomy and physiology to computer modelling - into a comprehensive theory of how the mouse uses its cortex to see. Following Allen's founding mandate for the institute, we will curate and publicly share all data and other resources (such as transgenic animals, probes and neuronal morphologies) for free, even before publication.

We believe that this initiative signals the arrival of large-scale science in a field that is populated by small groups. It will require rewarding the team, rather than a few lead investigators, for its collective effort. We envisage a time in the future when sophisticated, well-equipped brain observatories — 'mindscopes' - will complement the many academic neuroscience labs that will continue to make the bulk of discoveries.

Although the institute's project will focus on visual stimuli in mice, it will capture fundamental aspects of higher brain functions: perception, conscious awareness and decision-making, and how they lead to action. Once neuroscientists know the basic mechanisms in the mouse, they may start to understand more-complex forms of perception in other animals, including humans. In short, we believe that this project has the potential to revolutionize our understanding of the mammalian brain.

\section{VISION}

The brain observatories initiative focuses on the cerebral cortex - the proverbial 'grey matter' that is one of the hallmarks of mammals, and the seat of cognition and intelligence. For its size, the cortex is the most complex piece of organized matter in the known Universe. But given a grain of cortex, it is not easy to tell from which mammal it came. We all have the same basic hardware humans just happen to have a thousand times more of it than mice (see 'Man versus mouse').

The project will start with the anatomy of cortical neurons, using molecular techniques to count and catalogue the diverse array of cells that transmit information into and out of the visual cortex, as well as those that lie solely within the cortex. Then we will observe and measure the electrical activity of a sizeable fraction of those neurons to learn how the function of the cortex follows from its structure. It is a big question, but less daunting if one considers the cortex as being composed of many copies of a basic local circuit.

Studies of the human brain have been revolutionized by magnetic resonance
ONATURE.COM For more on the European Human Brain Project: go.nature.com/fjcdtt

\section{MAN VERSUS MOUSE}

Comparing the human and mouse brain reveals why mapping the mouse brain is easier.

\begin{tabular}{l|l|l|} 
& Human & Mouse \\
Mass of brain & 1,500 grams & 0.5 grams \\
Number of brain neurons & 86 billion & 70 million \\
Number of cortical neurons & 16 billion & 14 million \\
Fraction of cortex that is visual & $20 \%$ & $\sim 10 \%$ \\
Cortical regions involved in vision & 30 & 10 \\
Number of neurons in these areas & 5 billion & 1 million-2 million \\
Axons in optic nerve & 1 million & 45,000
\end{tabular}

imaging, which reveals brain regions activated in real time. However, the underlying signals are slow and fuzzy, and brain regions are not the building blocks of the mind - neurons, in their immense complexity, are the atoms of perception and thought.

Fortunately, scientists can now study individual mammalian neurons in living brains, by making neurons fluoresce using genetic engineering. Neurons can be made to emit flashes of light when they are active or, using the most recent optogenetics techniques, can be controlled with light (named Method of the Year in 2010 by Nature Methods ${ }^{1}$ ). In this way, researchers can transiently and reversibly control certain events in specific cell types at defined times. Perturbing the nervous system lets neuroscience move from correlation to causation, from observing that a given circuit is activated whenever the animal makes a decision to inferring that this circuit is necessary for decision-making.

Other technological advances enable neuroscientists to visualize every axon, dendrite and synapse in a circuit using threedimensional electron microscopy. Another observatory in the visual-brain programme is designed to record the electrical activity of thousands of neurons simultaneously. When all of these techniques are stitched together, one can imagine a full physiological and structural characterization of entire brain regions.

Finally, we will use all of this information to generate realistic and dynamic computer models of the mouse cortex and its associated structures, and use these to build theories of how the visual cortex behaves. The modelling facility will be next door to the labs so that collaborators can mingle easily. This should shorten the time between prediction and experimental validation, a virtuous circle that will be iterated until the model faithfully reproduces the data.

\section{A CALCULATED RISK}

Even though neuroscientists have spent the past 120 years uncovering the constitutive elements of the mammalian brain, we are far from understanding how any higher brain function comes about. Yet such knowledge is vital, especially because of the toll that nervous-system injuries and disorders take on individuals, families and society at large.

Although the challenge may seem daunting, we are not the first to propose such a huge undertaking. For example, the European Human Brain Project, under the leadership of Henry Markram at the Swiss Federal Institute of Technology in Lausanne, hopes to bring together some 150 principal investigators in 22 countries to create working computer models of the mouse, rat, monkey and human brains ${ }^{2}$. The brain observatories programme at the Allen Institute differs in that we are focusing on a single sense, in a single species, in one institution.

We know that such an expensive project will have critics: the resources required could fund hundreds of other projects, so why focus them in this way? Our response is that funding agencies are already spending billions of dollars on many smaller projects across all areas of biomedical research, and the Allen Institute wants to pioneer a new approach. We want to understand one piece of brain tissue by integrating knowledge across techniques and scales, rather than distributing the funds more widely.

There is a risk that this project will not work out as we anticipate, and that the various brain observatories - looking at anatomy, physiology and modelling, for example — will not synergize to form a sophisticated understanding of the mouse visual cortex. There is no guarantee that neuroscience is ready to become big science - but the only way to find out is to try.

Christof Koch is at the Allen Institute for Brain Science, Seattle, Washington 98103, USA, and at the California Institute of Technology, Pasadena, California 91125, USA. R. Clay Reid is at Harvard Medical School, Boston, Massachusetts 02115, USA, and will join the Allen Institute this summer. e-mail:christofk@alleninstitute.org

1. Nature Methods 8, 1 (2011).

2. Waldrop, M. M. Nature 482, 456-458 (2012).

3. Herculano-Houzel, S. Front. Hum. Neurosci. 3, 31 (2009).

4. Herculano-Houzel, S., Mota, B. \& Lent, R. Proc. Natl Acad. Sci. USA 103, 12138-12143 (2006).

5. Wang, Q. \& Burkhalter, A. J. Comp. Neurol. 502, 339-357 (2007). 